\title{
METODOLOGÍA PARA LA IMPLEMENTACIÓN DE LA PERSPECTIVA DE GÉNERO EN LA FACULTAD OBRERO CAMPESINA ${ }^{1}$
}

\section{METHODOLOGY FOR THE IMPLEMENTATION OF GENDER MAINSTREAMING IN THE FACULTY OF FARMWORKERS}

\author{
Noemis Gómez Estrada*
}

RESUMEN

\begin{abstract}
La presente investigación está encaminada a proponer una metodología para implementar la perspectiva de género en la Facultad Obrero Campesina, en el contexto del proceso de enseñanza-aprendizaje de la Historia de Cuba, basándose en el materialismo dialéctico e histórico como método científico general y fundamentada teóricamente en la perspectiva de género como eje transversal de la educación cubana y la teoría histórico cultural del desarrollo humano.
\end{abstract}

PALABRAS CLAVE: CUBA * HISTORIA * METODOLOGÍA * GÉNERO * EDUCACIÓN * APRENDIZAJE

ABSTRACT

This research aims to propose a methodology to implement gender mainstreaming in the Faculty of Farmworkers in the context of the teaching and learning of History of Cuba, based on dialectical and historical materialism as a general scientific method and theoretically grounded on gender as a central focus of Cuban education and cultural historical theory of human development.

KEYWORDS: CUBA * HISTORY * METHODOLOGY * GENDER * EDUCATION * APPRENTICESHIP

Universidad de Granma, Cuba.

ngomeze@udg.co.cu

1 La autora agradece a Dra. Yoenia Infante Cabrera y la Dra. Sonia Videaux Videaux, por los aportes y comentarios al presente documento. 


\section{INTRODUCCIÓN}

La metodología que se presenta, es una variante con carácter operativo que propicia la proyección metodológica de la enseñanza, en la que el profesor de forma planificada dirige el aprendizaje de sus estudiantes, para lo cual debe tener en cuenta sus características, posibilidades y los contenidos, así como, la realidad pedagógica.

Esta metodología tiene como objetivo el diseño de un sistema de acciones, actividades, métodos, procedimientos y medios de enseñanza para la implementación de la perspectiva de género en la asignatura de Historia de Cuba en la Facultad Obrera Campesina.

Por lo tanto, su elaboración parte del análisis de los objetivos de la asignatura de forma gradual, los objetivo formativos, así como, los contenidos y los niveles de desempeño cognitivo de los estudiantes. Es importante considerar en la aplicación de esta metodología, las características de los estudiantes en lo que respecta a la enseñanza de adultos.

La dirección del aprendizaje en la educación de jóvenes y adultos, es el punto convergente entre la diversidad de la población en particular y la diversidad de programas que atiende. Esta requiere la reconceptualización de métodos y procedimientos acorde con las nuevas exigencias sociales que se establecen, ya sean materiales o espirituales y el desarrollo vertiginoso de las nuevas tecnologías, de forma que siempre conduzca a la producción de nuevos conocimientos.

El surgimiento y evolución de la educación de adultos ha transitado diferentes etapas: génesis, fundación, diversificación e institución. Se ha correspondido con el desarrollo histórico cultural concreto del país, sin obviar las influencias externas, además se ha desarrollado asociada con las gestas emancipadoras y sobre la base de la formación de la nacionalidad y la identidad cultural. La voluntariedad política del Estado y el gobierno cubano ha garantizado los logros en este tipo de educación, sin exclusiones y con la participación popular.

De esta forma se reafirma la aplicación de la práctica de la coeducación y de la escuela abierta a la diversidad, lo cual garantiza el acceso a los centros de formación, en cualquiera de las especialidades $y$ profesiones que ofrece dicho sistema, donde se refleja la voluntad del ingreso, permanencia y el trabajo por la graduación con éxito para todos sin ningún condicionamiento por razón de sexo, raza, religión, grupo social o capacidad.

De acuerdo a la Red Regional de Innovaciones Educativas para América Latina y el Caribe (2000: 7), los adultos que se insertan en los centros de estudio habilitados al efecto, poseen características diversas e intereses y motivaciones que lo diferencian de los estudiantes de otras enseñanzas:

$\diamond \quad$ Las primeras actividades sociales de la persona adulta son atender a la familia $y$ el trabajo.

$\diamond \quad$ La composición sociocultural de los alumnos adultos $y$ sus niveles de partida de conocimientos son muy heterogéneas $y$ requieren de atención individualizada.

$\diamond \quad$ La actividad de aprendizaje de la educación para adultos está estrechamente vinculada a las motivaciones de naturaleza económica y social, con respuesta de continuidad de estudio.

$\diamond \quad$ El adulto siente como deber $y$ como derecho la necesidad de capacitarse en función de su realización individual y de su mejor participación en la sociedad en las esferas política, económica y social.

$\diamond \quad$ La educación de adultos adopta diversas formas; se desarrolla a través de una gran variedad de instituciones $y$ cursos que terminan en una labor de gestión diversa y compleja que obligan a una interpretación flexible, ya que atiende desde la labor de eliminación del analfabetismo y la complementación del ciclo primario hasta el nivel medio superior que articula con las universidades.

$\diamond \quad$ El adulto es un protagonista político $y$ social - cualesquiera que sean sus funciones $y$ tareas $-y$ como tal hay que tratarlo. Tiene la experiencia y la madurez necesaria para asumir cambios de conducta a través de un proceso de auto 
educación y modificación de conductas indeseables. Sus edades oscilan entre los 18 y 50 años, lo que los coloca como jóvenes en algunos casos y adultos en otros.

$\diamond \quad$ En su totalidad son trabajadores $y$ amas de casa, que por problemas personales, de salud u otras causas, se apartaron del sistema educacional por largo tiempo.

$\diamond \quad$ La educación de adultos en Cuba no se puede definir con un concepto unívoco, ni verla solo en la óptica de un subsistema educativo. Esto se fundamenta en la gran diversidad de proyectos que atiende $y$ en la heterogeneidad de sus contenidos, estrategias, métodos, entre otros. La diversidad poblacional que acude a sus disímiles alternativas y los escenarios donde se desarrolla, constituye un problema digno de análisis y atención para los docentes.

\section{DESARROLLO}

La metodología se sustenta en las concepciones más avanzadas de la teoría del aprendizaje, de la disciplina de la Pedagogía y de la Psicología de la personalidad, pues a través de la implementación de la perspectiva de género se puede incidir en la formación de los estudiantes, asignándosele una gran importancia a la relación entre lo cognitivo y lo afectivo.

Para implementar la metodología se establecen cuatro fases fundamentales:
A) Planificación
B) Orientación y Capacitación
C) Ejecución
D) Evaluación

\section{PRIMERA FASE}

\section{A) PLANIFICACIÓN}

El objetivo consiste en: planificar y orientar a los profesores los objetivos de cada fase, precisando la elaboración de la prueba pedagógica inicial que deben realizar los estudiantes $y$ valorar sus resultados, asimismo, a la encuesta aplicada a los profesores $y$ determinar las unidades $y$ sistemas de clases en la que le dará tratamiento a la perspectiva de género en las lecciones.

Las acciones a desarrollar con los profesores son:

1) Elaboración de orientaciones para la aplicación de la prueba pedagógica inicial a los estudiantes (anexo 3).

2) Desarrollar encuesta a los profesores seleccionados (anexo 1).

3) Análisis de los resultados de la encuesta aplicada a los profesores (anexo 2) y de la prueba pedagógica inicial que se aplicó a los estudiantes en las clases (anexo 4).

4) Determinar las unidades del programa $y$ el sistema de clases en los que se implementará la perspectiva de género.

5) Realización de la caracterización de los semestres a partir de un diagnóstico integral.

Teniendo en cuenta las condiciones concretas en que transcurre el proceso de enseñanza-aprendizaje, es necesario aplicar un diagnóstico integral que permita conocer la situación real de los profesores y estudiantes para implementar la perspectiva de género en la asignatura de Historia de Cuba.

Para la realización del diagnóstico integral, se han establecido indicadores con el fin de lograr una adecuada caracterización de los profesores $y$ los estudiantes.

En el diagnóstico a los profesores sobre conocimiento sobre la perspectiva de género, se debe tener en cuenta los siguientes elementos:

$\diamond \quad$ Que relacionen las bibliografías que han tenido la oportunidad de leer sobre las problemáticas de género.

$\diamond \quad$ Que expresen si su asignatura posee potencialidades para la implementación de la perspectiva de género.

$\diamond \quad$ Que expresen si se sienten preparados para implementar la perspectiva de género desde el contenido de su asignatura.

En el diagnóstico a los estudiantes se evaluaron los siguientes elementos: 
$\diamond \quad$ Dominio de los hechos históricos ocurridos en el Municipio de Buey Arriba de la provincia Granma en la Revolución de 1868 y 1895, así como la participación femenina en estos hechos.

$\diamond \quad$ Conocimiento de la actitud del gobierno de Estados Unidos frente al proceso revolucionario de los años 30.

$\diamond \quad$ Dominio del papel desempeñado por la mujer cubana en el despertar de la conciencia nacional antiimperialista de 1920-1925.

Los profesores deben conocer el modelo, para que con base en la realidad de sus estudiantes (el diagnóstico), poder implementar la propuesta y cumplir determinados requisitos para ser considerado un profesor preparado integralmente. Para ello deben conocer los objetivos formativos de la educación, poseer un nivel de preparación metodológica para enfrentar la actividad, tener disposición y actitud para desarrollar la actividad y tener dominio de la perspectiva de género.

\section{UNIDADES Y SISTEMAS DE CLASES DONDE SE IMPLEMENTARÁ LA PERSPECTIVA DE GÉNERO, EN EL PROCESO DE ENSEÑANZA-APRENDIZAJE DE LA ASIGNATURA DE HISTORIA DE CUBA}

El programa de Historia de Cuba de la Facultad Obrero Campesina está vigente desde el curso escolar 2004-2005. La implementación de la perspectiva de género constituye un eje transversal que atraviesa todo el proceso pedagógico en la escuela cubana, debido a la prioridad que confiere la temática para dar cumplimiento a los acuerdos asumidos por Cuba en la Conferencia de Beijing sobre la eliminación de todas las formas de discriminación y exclusión social.

Por sus potencialidades, el Programa de Historia de Cuba constituye una vía para lograr desde el análisis de los contenidos históricos, un adecuado tratamiento a la perspectiva de género, a través de la demostración de la participación de los hombres y mujeres en las luchas por la independencia nacional, lo que favorecerá el aprendizaje de la asignatura y la integración de todos y todas en el proyecto social cubano actual.

En la unidad 2, "La Revolución Cubana: la lucha del pueblo cubano contra el dominio colonial español (1868-1898)", del programa de Historia de Cuba del nivel medio superior para la Facultad Obrero Campesina, se estudia el papel de la mujer en la Guerra de Los Diez Años, la Tregua Fecunda y la Guerra Necesaria, contenidos que devienen en una fortaleza del sistema de clases para el tratamiento de la perspectiva género en el proceso de enseñanzaaprendizaje a través del estudio de las mujeres protagonistas de la historia de $\mathrm{Cuba}^{2}$, tales como: Ana Betancourt ${ }^{3}$, Mariana Grajales ${ }^{4}$ o de la historia local, como Rosa Castellanos ${ }^{5} y$ Adriana del Castillo ${ }^{6}$.

En la unidad 3 de este programa, "Las luchas del pueblo cubano por alcanzar su total independencia hasta 1935", se estudian los hechos y procesos más importantes del período, en los cuales se demuestran el despertar de la conciencia nacional $y$ antiimperialista en la sociedad cubana; en esta etapa importante se produjeron los congresos de mujeres en este

$2 \quad$ El movimiento nacional liberador cubano del siglo XIX estuvo matizado por el protagonismo social de las mujeres convertidas en soldados de la revolución al quedar al cuidado de los hijos, ancianos o alzarse también a la manigua, para que sus esposos pudieran combatir al enemigo colonial español. Su papel no quedó reducido a ser esposa o ama de casa, sino que también protagonizaron hechos históricos que demostraron su intransigencia revolucionaria y patriotismo.

3 Destacada independentista cubana y patriota de la ciudad de Camagüey. Proclamó la redención de la mujer cubana en la Asamblea de Guáimaro.

4 Destacada mujer que consagró la vida a la lucha por la independencia de Cuba. Reconocida por la educación que le dio a sus hijos, los Maceo, pro de la consecución de la libertad cubana.

5 Conocida como "La Bayamesa", fue una luchadora en las guerras de liberación nacional de Cuba y una hábil enfermera; organizó hospitales de campaña, y empuñó el machete y el fusil en varias incursiones guerreras.

6 Realizaba labores de enfermera junto a sus hermanas Lucila y Atala. Después de la quema de Bayamo, en enero de 1869, se marcha junto con su familia a la Sierra Maestra. 
país donde por primera vez se debatieron sus derechos, su programa de lucha y su proyección internacional.

Esta fase se evaluará a través de un taller donde se analicen los resultados del diagnóstico aplicado a los estudiantes (prueba pedagógica) $y$ de la encuesta aplicada a los profesores, para que se determinen las unidades y los sistemas de clases en los que se implementará la perspectiva de género.

\section{SEGUNDA FASE}

\section{B) ORIENTACIÓN Y CAPACITACIÓN}

El objetivo es orientar y capacitar a los profesores en la metodología para el tratamiento de la perspectiva de género en la asignatura de Historia de Cuba, con la finalidad de implementarla en el curso con los estudiantes.

En esta fase se efectuarán talleres con los profesores, que propicien la profundización en el estudio de la perspectiva de género, teniendo en cuenta las acciones a realizar, las actividades, los métodos, los procedimientos, las habilidades y las técnicas grupales participativas que motiven a los estudiantes $y$ a la vez propicie mayor unidad.

Se recomienda emplear métodos que propicien en los estudiantes un mejor nivel de desempeño, como los propuestos por Addine (2004):

1) El método expositivo problémico que facilitará al profesor desarrollar las capacidades de los estudiantes en todas sus potencialidades. Favorece el nivel de independencia, así como el empleo del debate, valoración y solución de situaciones problémicas.

2) El método heurístico o de búsqueda parcial permite mediante un adecuado sistema de preguntas, el diálogo con los estudiantes, el análisis y estudios de lecturas comentadas, realizar un aprendizaje en la que estudiante sea un protagonista histórico social vinculándolo con su vida personal y familiar.

3) El método investigativo ofrece múltiples posibilidades de aplicación, aún cuando exige un mayor nivel de complejidad de la actividad pedagógica. Desarrolla al máximo la actividad cognoscitiva y el pensamiento creador del estudiante. Si bien no requiere de la intervención abierta del educador, necesita de su orientación y control.

Por supuesto, la aplicación de estos métodos no implica que deban obviarse los métodos del nivel de reproducción, ya que el aprendizaje debe dirigirse de lo conocido a lo desconocido, de lo fácil a lo difícil. Para dar tratamiento a la diversidad, que es una característica típica de los estudiantes de la Facultad Obrera Campesina.

La evaluación se efectuará en el contexto de las actividades a través de talleres de forma parcial.

\section{TERCERA FASE}

\section{C) EJECUCIÓN}

En esta fase el objetivo es aplicar los profesores la metodología con los estudiantes por parte de los profesores, para implementar la perspectiva de género en el proceso de enseñanza-aprendizaje de la asignatura de Historia de Cuba de la Facultad Obrero Campesina.

\section{EJEMPLOS QUE ILUSTRAN COMO EJECUTAR LA METODOLOGÍA CON LOS ESTUDIANTES}

\section{Ejemplo 1}

Orientaciones a seguir para implementar este ejemplo

$\diamond \quad$ Objetivo del caso: Demostrar la potencialidad de los contenidos del programa de Historia de Cuba en el fortalecimiento de valores como la honestidad, la solidaridad $y$ el humanismo mediante el estudio de todos los contenidos del programa, pero haciendo énfasis en la Asamblea de Guáimaro y el papel de la mujer en la guerra.

$\diamond \quad$ Objetivo formativo general: Fortalecer el humanismo y la solidaridad en los 
estudiantes a través de la implementación de la perspectiva de género en los contenidos de la Asamblea de Guáimaro y el papel de la mujer en la guerra.

$\diamond \quad$ Método: heurístico o de búsqueda parcial.

$\diamond \quad$ Procedimientos: debate, diálogo, reflexión, análisis y síntesis.

$\diamond \quad$ Medios de Enseñanza: libro de texto de Historia de Cuba del Nivel Medio Superior $y$ softwares educativos como Todo de Cuba (Historia) y Colección Futuro (Convicciones), pizarra y fichas de contenido.

$\diamond \quad$ Técnicas participativas a utilizar: lluvias de ideas, mesa redonda, discordar y concordar, creación de poemas grupales, técnica del enrejado para la formación de los equipos y el debate de las actividades.

$\diamond \quad$ Habilidad: explicar.

Se implementará en la unidad 2 "La Revolución Cubana: la lucha del pueblo cubano contra el dominio colonial español (1868-1898)" de la asignatura Historia de Cuba (encuentro 5). Se seleccionará el contenido de la temática sobre "El desarrollo de la lucha por la independencia de Cuba en la Guerra de los Diez Años. Labor revolucionaria de las mujeres". El objetivo consistirá en explicar el desarrollo y radicalización del proceso revolucionario en los primeros años de la guerra grande, las primeras manifestaciones de unidad y el papel desempeñado por la mujer en la guerra para fortalecer los valores de independentismo, solidaridad y humanismo en los estudiantes.

Acciones a realizar:

1. Interpretar el objeto o información: seleccionar las características fundamentales del desarrollo y radicalización del proceso revolucionario en los primeros años de la guerra grande.

2. Argumentar los juicios de partida: identificar los hechos fundamentales que denotan la radicalización del proceso revolucionario.

3. Establecer las interrelaciones de los argumentos: estudiar las figuras históricas de Ignacio Agramonte y Máximo Gómez, así como, su papel desempeñado en la radicalización del proceso revolucionario.

4. Ordenar lógicamente las interrelaciones encontradas: analizar los hechos mencionados en los puntos anteriores, así como, las manifestaciones de unidad y el papel desempeñado por la mujer en la guerra.

5. Exponer ordenadamente los juicios y razonamientos: presentar juicios del contenido, a partir de las ideas rectoras del proceso de enseñanza-aprendizaje de la asignatura de Historia de Cuba.

\section{Actividad 1}

¿Qué hecho histórico ocurrió el 10 de abril de 1869 ?

¿Cuáles fueron los principales acuerdos tomados en esta asamblea?

¿Cuál fue la posición de la mujer cubana ante la importante asamblea desarrollada? $¿$ Consideras correcto que existan igualdad de derechos para los hombres y las mujeres? ¿Por qué?

¿Cómo se manifestaron los roles de género en la asamblea desarrollada?

\section{Actividad 2}

En las primeras décadas del siglo xix, la mujer no podía tener participación activa en la vida social cubana ¿Por qué?

Tenga en cuenta los conocimientos que posee de la historia de Cuba, ¿qué evolución tuvo el rol de la mujer cubana en el propio siglo xix en la lucha por la causa de la Revolución? Cite ejemplos de esas mujeres que responden a esta caracterización. ¿Qué opina de esas mujeres?

¿Conoce alguna canción de la Nueva Trova que hable de un gran hombre de la guerra y su esposa? Explique. ¿Puede cantar algún fragmento?

La historia de nuestro país y sobre todo, la más reciente, muestra la existencia de mujeres que se han destacado en las esferas política, cultural, científica y deportiva. Mencione algunas de ellas. 
Realice una comparación entre esas mujeres $y$ las de principios del siglo XIX teniendo en cuenta: el tratamiento que les da la sociedad, la situación con respecto al hombre $y$ el papel que desempeñan en la sociedad.

Consultar además de la bibliografía recomendada, el software educativo Colección Futuro (Convicciones), Hechos y Realizaciones Concretas y Todo de Cuba (Historia).

La evaluación se efectuará de forma parcial, con la revisión de las actividades interencuentros $y$ en los aportes que realice el estudiante durante el curso.

\section{Ejemplo 2}

Orientaciones a seguir para implementar este ejemplo

$\diamond \quad$ Objetivo del caso: Demostrar la potencialidad de los contenidos del programa de Historia de Cuba en el fortalecimiento de valores como la honestidad, solidaridad $y$ humanismo mediante el estudio de todos los contenidos del programa pero haciendo énfasis en el Primer y Segundo Congreso de las Mujeres.

$\diamond \quad$ Objetivo formativo general: Fortalecer el humanismo y la solidaridad en los estudiantes, a través de la implementación de la perspectiva de género en los contenidos del Primer y Segundo Congreso de las Mujeres.

$\diamond \quad$ Método: heurístico o de búsqueda parcial.

$\diamond$ Procedimientos: debate, diálogo, reflexión, análisis y síntesis.

$\diamond \quad$ Medios de enseñanza: libro de texto de Historia de Cuba del Nivel Medio Superior, software educativos como Todo de Cuba (Historia) y Colección Futuro (Convicciones), pizarra y fichas de contenido.

$\diamond \quad$ Técnicas participativas a utilizar: lluvias de ideas, mesa redonda, paneles, discordar y concordar, creación de poemas grupales, técnica del enrejado para la formación de los equipos y el debate de las actividades.

$\diamond \quad$ Habilidades: explicar.

Se seleccionará la unidad 3 "Las luchas del pueblo cubano por alcanzar su total independencia hasta 1935", de la asignatura Historia de Cuba, con el contenido del tema "El auge de la conciencia nacional y antiimperialista en el movimiento revolucionario cubano hasta 1935".

Acciones a realizar:

1. Interpretar el objeto o información: seleccionar las características fundamentales del desarrollo y radicalización del proceso revolucionario de 1929-1935.

2. Argumentar los juicios de partida: identificar los hechos fundamentales que denotan la radicalización del proceso revolucionario.

3. Establecer las interrelaciones de los argumentos: estudiar la situación de la economía cubana de 1929-1933, para analizar las posiciones de las diferentes organizaciones ante la dictadura del gobierno de Machado y la actitud hostil del gobierno de eevu ante la independencia de Cuba con la mediación de Summer Wells.

4. Ordenar lógicamente las interrelaciones encontradas: analizar los hechos mencionados en los puntos anteriores, así como, el papel desempeñado por la mujer en este período.

5. Exponer ordenadamente los juicios y razonamientos: presentar juicios del contenido, a partir de las ideas rectoras del proceso de enseñanza-aprendizaje de la asignatura de Historia de Cuba.

Actividad 1 (actividades interencuentros)

1. En abril de 1923 se celebró el Primer Congreso Nacional de Mujeres:

- ¿ ¿Cuáles fueron los principales acuerdos tomados en este congreso?

- ¿ ¿Cuál fue su significación histórica? 
- ¿Por qué no participaron en este congreso las mujeres obreras y las mujeres negras?

- ¿Cuál fue el tratamiento que se les dio a las problemáticas de género en este congreso?

2. Respecto al Segundo Congreso de las Mujeres:

- $\quad$ ¿Cuáles fueron las principales problemáticas discutidas?

- ¿ ¿Cuál fue la significación histórica de este congreso?

- $\quad$ ¿Cuál fue el tratamiento que se les dio a las problemáticas de género en este Congreso?

La evaluación se efectuará de forma parcial, con la revisión de las actividades interencuentros a través de un panel.

\section{Ejemplo 3}

Orientaciones a seguir para implementar este ejemplo:

$\diamond \quad$ Objetivo del caso: Demostrar la potencialidad de los contenidos del programa de Historia de Cuba en el fortalecimiento de valores como la honestidad, la solidaridad y el humanismo mediante el estudio de todos los contenidos del programa, haciendo énfasis en el papel desempeñado por la mujer cubana en la última etapa de las luchas por la independencia de Cuba.

$\diamond \quad$ Objetivo formativo general: Fortalecer el humanismo y la solidaridad en los estudiantes, a través de la implementación de la perspectiva de género en los contenidos de Asalto a los Cuarteles Moncada y Carlos Manuel de Céspedes, la prisión de los moncadistas y la Constitución del Movimiento 26 de Julio.

$\diamond \quad$ Método: exposición heurística o búsqueda parcial, investigación.

$\diamond \quad$ Procedimientos: debate, diálogo, reflexión, análisis y síntesis.

$\diamond \quad$ Medios de Enseñanza: libro de texto de Historia de Cuba del Nivel Medio Superior, software educativos como
Todo de Cuba (Historia) y Colección Futuro (Convicciones), pizarra y fichas de contenido.

$\diamond \quad$ Técnicas participativas a utilizar: lluvias de ideas, mesa redonda, discordar y concordar, elaboración de poemas colectivos, paneles, entre otras.

$\diamond \quad$ Habilidad: valoración.

Se implementará en la unidad 5 "El período decisivo de las luchas revolucionarias de nuestro pueblo (1953-1958)", de la asignatura Historia de Cuba, con el contenido del tema "El papel de la mujer cubana en la última etapa de nuestras luchas por la independencia cuando se produjeron los Asaltos a los Cuarteles Moncada y Carlos Manuel de Céspedes". El objetivo consiste en valorar el papel de la mujer cubana en la última etapa de las luchas por la independencia.

\section{Acciones a realizar:}

1. Caracterizar el objeto de la crítica: analizar las características fundamentales de los Asaltos a los Cuarteles Moncada y Carlos Manuel de Céspedes.

2. Establecer los criterios de valorización: identificar los hechos fundamentales que denotan la nueva estrategia revolucionaria diseñada por Fidel Castro.

3. Comparar el objeto con los criterios de valor establecidos: estudiar la significación histórica de los Asaltos a los Cuarteles Moncada y Carlos Manuel de Céspedes, la prisión de los moncadistas y la constitución del movimiento 26 de julio.

4. Elaborar los juicios de valor acerca del objeto con el análisis de los hechos: analizar los puntos anteriores, así como, la labor revolucionaria desarrollada por la mujer en el período.

Actividad 1 (actividades interencuentros)

¿Cuáles fueron las mujeres cubanas que participaron en los Asaltos a los Cuarteles Moncada y Carlos Manuel de Céspedes? 
- ¿Cómo valorar su actitud ante las tensiones emocionales sufridas?

- ¿QQué actividades desempeñaron las mujeres cuando los sobrevivientes del Moncada estaban en el exilio?

- Valore las acciones fundamentales desarrolladas por las mujeres en la lucha clandestina y guerrillera de 1953-1959.

- ¿Cuáles fueron las féminas que más se destacaron en la lucha clandestina y guerrillera en su localidad? Valore su actividad revolucionaria.

La evaluación se efectuará de forma parcial, con la revisión de las actividades interencuentros, a través de la exposición de los equipos.

\section{CUARTA FASE}

\section{D) EVALUACIÓN}

Objetivo: Definir la evaluación sobre la base del conocimiento alcanzado, con la implementación de la metodología para el tratamiento de la perspectiva de género en los contenidos de la asignatura de Historia de Cuba.

La misma puede ser controlada no solo a través de la asignatura, sino en el espacio dedicado a las actividades extradocentes y extraescolares en las que participarán los estudiantes.

La evaluación puede ser cuantitativa, cualitativa o combinada, incluyendo la autoevaluación y la coevaluación. Pueden utilizarse técnicas participativas, de acuerdo con la forma de evaluación planificada. Se pueden tomar en consideración los siguientes aspectos:

1. Los conocimientos.

2. Nivel de utilización de las categorías esenciales de la perspectiva de género en el discurso oral.

3. Trabajos investigativos.

4. Actividades políticas y patrióticas con la expresión oral y escrita.

5. El desarrollo de actitudes y sentimientos humanistas en los educandos, así como, el fomento de sentimientos, valores $y$ normas de conducta positivos.
6. La promoción de la educación ciudadana para que en sus hogares, centros laborales $y$ comunidad desarrollen una actitud responsable.

La evaluación se efectuará a través de comprobaciones escritas a los estudiantes, desempeño en la clase, escuela y comunidad, así como, participación en talleres, seminarios y mesas redondas.

La realización de las evaluaciones permitirá un mayor aprendizaje en la asignatura de Historia de Cuba con una perspectiva de género, formación de valores (patriotismo, antimperialismo, humanismo, la responsabilidad y el internacionalismo), transformación de actitudes en modos de actuación positivos e integración de los conocimientos para resolver problemas de la vida práctica en el hogar, centro laboral o comunidad.

\section{CONCLUSIONES}

La implementación de la perspectiva de género en el programa de Historia de Cuba en la Facultad Obrero Campesina constituye actualmente una prioridad que se debe incluir en el proceso de enseñanza- aprendizaje de la asignatura, las relaciones de empoderamiento entre los géneros, la discriminación de diferentes grupos, las clases sociales y la historia de hombres y mujeres integradas como parte del contenido histórico nacional y local, que deben aprender los estudiantes.

El diseño de la metodología propuesta permite su configuración a partir de cuatro fases contentivas de ejemplos que ilustran como implementar la propuesta en la práctica escolar, contribuyendo a la transformación de los modos de comportamiento relacionados con el género.

Los métodos de exposición problémica, búsqueda parcial e investigativo dinamizan la dirección del aprendizaje de la asignatura de Historia de Cuba desde una perspectiva de género. La metodología que se propone como vía para implementar la perspectiva de género en el proceso de enseñanza-aprendizaje de esta asignatura, a través de sus etapas, está dirigida a garantizar un aprendizaje creativo, flexible, reflexivo y crítico en los estudiantes de la Facultad Obrero Campesina. 
BIBLIOGRAFÍA

LIBROS

Acebo, Waldo. Apuntes para una metodología de la enseñanza de la historia local en su vinculación con la historia patria. La Habana, Cuba: Ed. Pueblo y Educación, 1991.

Addine Fernández, Fátima. Didáctica teoría y práctica. La Habana, Cuba: Editorial Pueblo y Educación, 2004.

Álvarez, Carlos Manuel. La escuela en la vida. La Habana, Cuba: Editorial Pueblo y Educación, 1993.

Álvarez, Rita Marina. Hacia un currículum integral y contextualizado. Tegucigalpa, Honduras: Ed. Universitaria, 1997.

Álvarez, Rita M. y Díaz, Horacio. Metodología de la enseñanza de la Historia. Tomo I y II. La Habana, Cuba: Ed. Pueblo y Educación, 1978.

Arjona, Martha. Patrimonio cultural $e$ identidad. La Habana, Cuba: Letras Cubanas, 1986.

Aróstegui, Julio. La investigación histórica: teoría y método. Barcelona, España: Crítica, 1995.

Baeza, Cristina. Modelo teórico para la identidad cultural. La Habana, Cuba: Ed. Centro de Investigación para la Cultura Cubana "Juan Marinello", 1996.

Báxter, Esther; Amador, Amelia y Bonet, Mirtha. La escuela y el problema de la formación del hombre. La Habana, Cuba: Instituto Central de Ciencias Pedagógicas, 1994.

Benejam, Pilar y Pagés, Joan (coord). Enseñar $y$ aprender ciencias sociales, Geografía e Historia en la educación secundaria. Barcelona, España: Institut de Ciències de l'Educació (ICE) de la Universidad de Barcelona, 1997.

Benítez, María Elena. La familia cubana en la segunda mitad del siglo xx. La Habana, Cuba: Ed. Ciencias Sociales, 2003.

Bermúdez, Raquel y Pérez, Lorenzo M. Aprendizaje formativo y crecimiento personal. La Habana, Cuba: Ed. Pueblo y Educación, 2004.
Blanco, Antonio. Introducción a la Sociología de la educación. La Habana, Cuba: Facultad de Ciencias de la Educación, 2000.

Carretero, Mario. Construir y enseñar. Las ciencias sociales y la Historia. Buenos Aires, Argentina: Aique, 1995.

Casanova, Julián. La Historia Social y los historiadores. Barcelona, España: Crítica, 1991.

Castellanos, Beatriz. Sexualidad humana, personalidad y educación. La Habana, Cuba: Ed Científico Técnica, 1995.

Castellanos, Doris. Teoría psicológica de aprendizaje. La Habana, Cuba: Ediciones CIFPOE, 1994.

Castellanos, Doris. La comprensión de los procesos de aprendizaje. Apuntes para un marco conceptual. La Habana, Cuba: Centro de Estudios EducacionalesInstituto Superior Pedagógico Enrique José Varona, 1999.

Castellanos, Doris; Castellanos, Beatriz; Llivina, Miguel Jorge; Silverio, Mercedes; Reinoso, Carmen y García, Celina. Aprender y enseñar en la escuela. Una concepción desarrolladora. La Habana, Cuba: Ed. Pueblo y Educación, 2005.

Díaz, Horacio (comp.). Enseñanza de la Historia. Selección de lecturas. La Habana, Cuba: Pueblo y Educación, 2002.

Díaz, Horacio. Acerca de la clasificación de los medios de enseñanza de la Historia. La Habana, Cuba: Pueblo y Educación, 1989.

Díaz, Horacio. Aprendiendo Historia en el Museo. La Habana, Cuba: Ed. Pueblo y Educación, 1991.

Engels, Federico. "Del socialismo utópico al socialismo científico". Obras escogidas en dos tomos de Carlos Marx y Federico Engels. Tomo II. Moscú, Rusia: Editorial Progreso, 1955.

Engels, Federico. "El papel del trabajo en la transformación del mono en hombre". Obras Escogidas. Tomo I. Moscú, Rusia: Editorial Progreso, 1978.

Engels, Federico. "Ludwing Feuerbach y el fin de la filosofía clásica alemana". Obras 
escogidas en dos tomos de Carlos Marx y Federico Engels. Tomo II. Moscú, Rusia: Editorial Progreso, 1955.

Engels, Federico. El origen de la familia, la propiedad privada y el Estado. La Habana, Cuba: Editorial Prensa Libre, 1963.

García de León, María Antonia; de la Fuente, Gloria y Ortega, Félix (eds.). Sociología de la educación. Barcelona, España: Barcanova, 1993.

García, Gaspar. Glosas sobre el libro de Lenin "materialismo y empiriocriticismo". La Habana, Cuba: Editorial Academia, 1979.

García, María del Carmen. "El feminismo contemporáneo. Una mirada desde México". Historia de las Mujeres en América Latina. Juan Andreo García y Sara Beatriz Guardia (comp. y ed.). España: Universidad de Murcia, 2002. En: $<$ http://www.um.es/estructura/unidades/ u-igualdad/intranet/docs/historia-delas-mujeres-en-america-latina.pdf > [Consultado el 15 de junio de 2011].

González, Alicia. Sexualidad y géneros alternativas para su educación en el siglo xхı. La Habana, Cuba: Ed Científico Técnica, 2003.

González, Fernando. Algunas cuestiones teóricas y metodológicas sobre el estudio de la personalidad. La Habana, Cuba: Ed. Pueblo y Educación, 1982.

González, Julio C. En busca de un espacio: Historia de las Mujeres en Cuba. La Habana, Cuba: Ed Ciencias Sociales, 2002.

González, María del Carmen. La enseñanza de la Historia en el nivel medio. Situación, tendencias e innovaciones. Madrid: Marcial Ponsm, 1996.

González, Viviana. Psicología para educadores. La Habana, Cuba: Ed. Pueblo y Educación, 2001.

Marx, K. y Engels, F. "La ideología alemana". Obras escogidas. Moscú, Rusia: Editorial Progreso, 1978.

\section{PUBLICACIONES PERIÓDICAS}

Aguilera, Manuela. "Violencia y género". Crítica 933(56). España. Fundación Castroverde, marzo 2006.
Caamaño, Victor Manuel. "Sexualidad y género en la educación transversal". La tarea. Revista de Educación y Cultura 15. México: Sindicato Nacional de Trabajadores de la Educación, 2001. En $<$ http://www.latarea.com.mx/articu/ articu15/caaman15.htm> [consultado el 15 de junio de 2011].

Durán, María. "Los usos del tiempo y el cambio social”. Crítica 933 (56). España. Fundación Castroverde, marzo 2006.

García, Alejandro. "Apuntes cubanos sobre la Historia y sus métodos". Revista Contracorriente 5 (2). Habana, Cuba. Ministerio de cultura y La Unión de escritores $y$ artistas de Cuba. Julioagosto-septiembre, 1996: 6-13.

TESIS

Abreu, Omar. "Modelo didáctico para la introducción del enfoque martiano acerca del anexionismo en la formación del profesor de Humanidades del preuniversitario". [Tesis de Doctorado en Ciencias Pedagógicas]. Cuba: Universidad de Pinar del Río, 2006.

Arteaga, Frank. "Propuesta didáctica para el funcionamiento de las Aulas Martianas en el noveno grado de la enseñanza media básica". [Tesis de Doctorado en Ciencias Pedagógicas]. Las Tunas, Cuba: Universidad de Ciencias Pedagógicas "Pepito Tey", 2002.

Bao, Liuska. "Una concepción didáctica dirigida a desarrollar los intereses cognoscitivos, profesionales y sociales de los estudiantes para el estudio de la Historia de Cuba en la educación superior". [Tesis de Doctorado en Ciencias Pedagógicas]. Holguín, Cuba: Universidad de Ciencias Pedagógicas "Pepito Tey", 2004.

Castillo, Niurca. "Metodología para la Educación de la Sexualidad de los adolescentes en el contexto de las actividades extradocentes del nuevo modelo educativo de secundaria básica". [Tesis de Doctorado en Ciencias Pedagógicas]. Cuba: Universidad de Pinar del Río, 2005. 
Cortina, Víctor. "El diagnóstico pedagógico en el proceso formativo del profesional de la educación en condiciones de universalización". [Tesis de Doctorado en Ciencias Pedagógicas]. Las Tunas, Cuba: Universidad de Ciencias Pedagógicas "Pepito Tey", 2005.

González, Ernesto. "La identidad cultural universitaria, en función de la contextualización de los procesos de pertinencia e impacto social de las universidades". [Tesis de Doctorado en Ciencias Pedagógicas]. Cuba: Universidad de Granma, 2011.

González, Pedro Luis. "Modelo de capacitación desde la Historia Contemporánea para los profesores en ejercicio de Secundaria Básica en Pinar del Río". [Tesis de Doctorado en Ciencias Pedagógicas]. Cuba: Universidad de Pinar del Río, 2005.

Infante, Yoenia. "¿Historia familiar en el currículo de Secundaria Básica?". [Trabajo de Diplomado en Educación en la Especialidad de Marxismo- Leninismo e Historia]. Las Tunas, Cuba: Universidad de Ciencias Pedagógicas "Pepito Tey", 2000.

Infante, Yoenia. "Concepción didáctica para el tratamiento a la historia de las mujeres en la educación secundaria básica”. [Tesis de Doctorado en Ciencias Pedagógicas]. Las Tunas, Cuba: Universidad de Ciencias Pedagógicas "Pepito Tey", 2008.

López, Joel. "El desarrollo de la identidad cultural en estudiantes de preuniversitario". [Tesis de Doctorado en Ciencias Pedagógicas]. Cuba: Universidad de Granma, 2009.

Videaux, Sonia. "Reflexiones teóricas y empíricas en torno al papel de la mujer en la familia granmense". [Tesis de Doctorado en Ciencias Filosóficas]. Cuba: Universidad de Granma, 1999.

\section{TEXTOS ELECTRÓNICOS}

Castaño, José. Gender. 2001. En <http://www. europarl.europa.eu> [consultado el 10 de marzo de 2001].

Cuartero, Santos. Aportaciones al estudio en la secundaria de la mujer en el
Sistema Feudal. España: Dialnet, 2006. En: <dialnet.unirioja.es/descarga/ articulo/1454152.pdf $>$ [consultado el 12 de mayo de 2012].

EcuRed. Biografía de Ana Betancour. Cuba, 2015. En: <http://www.ecured.cu/index. php/Ana_Betancourt> [consultado el 28 de julio de 2015].

EcuRed. Biografía de Mariana Grajales. Cuba, 2015. En: <http://www.ecured.cu/index. php/Mariana_Grajales> [consultado el 28 de julio de 2015].

EcuRed. Biografía de Rosa Castellanos. Cuba, 2015. En: <http://www.ecured.cu/index. php/Rosa_Castellanos> [consultado el 28 de julio de 2015].

EcuRed. Biografía de Adriana del Castillo. Cuba, 2015. En: <http://www. ecured.cu/index.php/Adriana_del_ Castillo_V\%C3\%A1zquez> [consultado el 28 de julio de 2015].

González, Julio C. Feminismo $y$ masculinidad ¿Mujeres contra hombres? 2004. En: <https://www. euskadi.eus/contenidos/informacion/ material/es_gizonduz/adjuntos/femini smoymasculinidad;\%C2\%BFmujeresco ntrahombres.pdf $>$ [consultado el 12 de mayo de 2007].

González, Julio C. Género y masculinidad en Cuba ¿el otro lado de una Historia? Cuba: Cuba Literaria y Portal de Literatura Cubana, 2005. En: <www. cubaliteraria.cu/articulo.php?idarticulo= 11293\&idseccion=25> [consultado el 12 de mayo de 2010].

OTROS

Álvarez, Mayda. Género, familia y relaciones de poder. CD-Rоoм, 2005.

Arés, Patricia. "Las investigaciones sobre la familia cubana desde la Psicología”. Conferencia. Conferencia Iberoamericana: las familias y las culturas. CD-Room, 2005.

Castellanos, Doris. "Educación, aprendizaje $y$ desarrollo". Ponencia. Congreso Internacional Pedagogía. La Habana, Cuba. 2001. 
Castellanos, Doris y Grueiro, Irene. "Enseñanza $y$ estrategias de aprendizaje: los caminos del aprendizaje autorregulado". Ponencia. Congreso Internacional Pedagogía. La Habana, Cuba. 1999.

De Miguel Álvarez, Ana. Los feminismos a través de la Historia. s/f. En: <http:// webserver.rcp.net> [Consultado el 02 de marzo de 2007].

Díaz, Horacio. "A propósito de la enseñanza de la Historia". Ponencia. I Seminario Nacional para Educadores. La Habana, Cuba: Ed. Pueblo y Educación, 2000.

Díaz, Horacio. "Dirección del proceso de enseñanza aprendizaje de las asignaturas priorizadas: Historia”. Ponencia. V Seminario Nacional para Educadores. La Habana, Cuba: Ed. Pueblo y Educación, 2004.

Díaz, Horacio. "Enseñanza de la Historia". Ponencia. vII Seminario Nacional para Educadores. La Habana, Cuba: Ed. Pueblo y Educación, 2007.

Domínguez, Consuelo y Estepa, Jesús. "La igualdad de mujeres y hombres más allá de la escuela: estrategias de aprendizajes". Ponencia. Simposio Internacional de Didáctica de las Ciencias Sociales. 2004. En: < http:// rabida.uhu.es/dspace/bitstream/ handle/10272/9771/La_igualdad_de_ mujeres_y_hombres.pdf? sequence $=2>$ [consultado el 11 de mayo de 2006].
Infante, Yoenia. "La dirección del aprendizaje en el nuevo modelo de la Secundaria Básica”. Evento Internacional Pedagogía. CD-ROом, 2005.

Infante, Yoenia. Mujer-familia ¿Problemas o soluciones? $\mathrm{CD}-\mathrm{Room}, 2005$.

Infante, Yoenia. "Enseñanza de la Historia en el nuevo modelo de la Secundaria Básica". Ponencia. Congreso Internacional Pedagogía. cD-Roом, 2007.

Infante, Yoenia. "Metodología para el tratamiento a los contenidos de la historia de las mujeres en el proceso de enseñanza-aprendizaje de la Historia de Cuba". Ponencia. Congreso Nacional de Historia. CD-Room, 2008.

Infante, Yoenia. "Propuestas didácticas para mejorar el proceso de enseñanza aprendizaje de la Historia desde el trabajo colectivo del Proyecto Cliodidáctica”. Ponencia. Congreso Provincial Pedagogía, 2009.

Infante, Yoenia. Reflexiones sobre la importancia de la historia de la mujer en la educación de la personalidad de los adolescentes. CD-Rоом, 2005.

Infante, Yoenia. La relación historiografía, historia de las mujeres y enseñanza de la historia. 2006.

Microsoft Corporation. Enciclopedia Microsoft Encarta, 2010. 


\section{ANEXO 1}

\section{ENCUESTA INICIAL}

Objetivo: constatar los conocimientos que poseen los profesores acerca de la perspectiva de género.

\section{CUESTIONARIO}

1. ¿Qué es el género?

2. ¿Cuáles son las categorías de la perspectiva de género?

a) Defina cada una de ellas.

3. ¿Cuáles son las problemáticas principales de la perspectiva de género en la Facultad Obrero

Campesina?

4. ¿Qué acciones realizas en las clases de Historia de Cuba para potenciarlo?

5. ¿Consideras que la perspectiva de género es un eje transversal o un programa director?

Sí ___ o No ___. ¿Por qué? 


\section{ANEXO 2}

RESULTADOS DE LA APLICACIÓN DE LA ENCUESTA INICIAL.

Resultados de la encuesta inicial realizada al docente.

\begin{tabular}{|c|c|c|c|c|c|}
\hline \multicolumn{6}{|c|}{ Categorías } \\
\hline B & $\%$ & R & $\%$ & M & $\%$ \\
\hline- & - & 1 & 50 & 1 & 50 \\
\hline
\end{tabular}

\section{ANEXO 3}

\section{PRUEBA PEDAGÓGICA INICIAL.}

Objetivo: constatar los conocimientos que poseen los estudiantes acerca de la Historia de Cuba de (1868 - 1935).

\section{CUESTIONARIO}

1. Mencione los hechos históricos ocurridos en el municipio durante la Revolución de 1868 y 1895.

a) ¿Qué nombres de mambises y mambisas no se deben olvidar?

2. De las luchas contra el dominio colonial español identifica los siguientes hechos históricos:
a) Incendio de Bayamo.
b) Fin de la Guerra de los Diez Años.
c) Reunión de la Mejorana.

3. Caracteriza la Asamblea de Guáimaro.

a) ¿Qué papel desempeñó la mujer en este importante momento de unidad?

4. Demuestra con 4 elementos la actitud del gobierno de Estados Unidos frente al proceso revolucionario de los años 30.

5. Valore el papel desempeñado por la mujer cubana en el despertar de la conciencia nacional antiimperialista de 1920 - 1925. 


\section{ANEXO 4}

RESULTADOS DE LA APLICACIÓN DE LA PRUEBA PEDAGÓGICA INICIAL

\begin{tabular}{|c|c|c|c|c|c|c|}
\hline \multirow{3}{*}{ Prueba } & \multirow{3}{*}{ Pioneros } & \multirow{3}{*}{ Categoría } & \multirow{3}{*}{$\%$} & \multicolumn{3}{|c|}{ Niveles } \\
\hline & & & & \multirow{2}{*}{ I } & & \\
\hline & & & & & II & III \\
\hline \multirow{3}{*}{ Inicial } & 12 & B & 76.6 & 37 & 5 & 3 \\
\hline & 33 & $\mathrm{R}$ & 73.3 & - & - & - \\
\hline & - & M & - & & - & - \\
\hline Total & 45 & & 100 & - & & \\
\hline
\end{tabular}

Fecha de ingreso: 24/ 01/2014 Fecha de aprobación: 15/10/2014 